\title{
Thyroid Control over Biomembranes: VI. Lipids in Liver Mitochondria and Microsomes of Hypothyroid Rats
}

\author{
F.L. HOCHa,b, C. SUBRAMANIANd , G.A. DHOPESHWARKARe,d, and J.F. MEAD ${ }^{\mathrm{d}, \mathrm{d}}$
}

\begin{abstract}
The lipids of liver mitochondria prepared from normal rats and from rats made hypothyroid by thy roidectomy and injection with ${ }^{131} \mathrm{INa}$ contained similar amounts, per $\mathrm{mg}$ protein, of total lipids, phospholipids, neutral lipids and lipid phosphorus. Hypothyroidism caused a doubling of the relative amounts of mitochondrial cardiolipins (CL; to $20.5 \%$ of the phospholipid P) and an accompanying trend (al though statistically not significant) toward decreased amounts of both phosphatidylcholines (PC) and phosphatidylserines (PS), with phosphatidylethanolamines (PE) remaining unchanged. The pattern of elevated 18:2 fatty acyl content and depleted 20:4 acyl groups of the mitochondrial phospholipids of hypothyroid preparations was reflected to varying degrees in the resolved phospholipids, with PC showing greater degrees of abnormality than PE, and CL showing none. Hypothyroidism produced the same abnormal pattern of fatty acyl distributions in liver microsomal total lipids as was found in the mitochondria. Hypothyroid rats, when killed $6 \mathrm{hr}$ after injection of $\left[1{ }^{14} \mathrm{C}\right]$ labeled linoleate, showed the following abnormalities: the liver incorporated less label into lipids, and converted 18:2 not exclusively to $20: 4$ (as normals do) but instead incorporated the label mainly into saturated fatty acids. These data, together with the known decrease in $\beta$-oxidation, suggest that hypothyroidism involves possible defective step(s) in the conversion of $18: 2$ to $20: 4$.
\end{abstract}

\section{INTRODUCTION}

The slowed rate of ADP phosphorylation in the liver mitochondria of hypothyroid rats, when measured at $25-30 \mathrm{C}$, is one aspect of an abnormal velocity-temperature relationship (1-3). Because Arrhenius plot profiles of membrane-dependent catalytic and transport systems alter when membrane unsaturated fatty acyl contents are manipulated $(4,5)$, we measured the phospholipid fatty acyl groups in liver mitochondria $(2,3)$. Hypothyroidism increased the contents of linoleoyl (18:2) and decreased arachidonoyl $(20: 4)$ acyl groups and because of the major contribution of $20: 4$ to the unsaturation index $(\Sigma[\%$ fatty acyl group $\times$ number of unsaturated bonds]) decreased overall unsaturation. A similar correlation has been reported between the abnormal Arrhenius profile of State 3 respiration (succinate) and

${ }^{a}$ These studies were initiated during a leave at the University of California, Los Angeles.

b Departments of Internal Medicine and Biological Chemistry, University of Michigan Medical School, 7696 Kresge Building, Ann Arbor, MI 48109.

${ }^{c}$ Department of Biological Chemistry and School of Public Health, University of California at Los Angeles.

dLaboratory of Nuclear Medicine and Radiation Biology, University of California at Los Angeles.

e Division of Environmental and Nutritional Sciences, School of Public Health, University of California at Los Angeles. altered unsaturation (6).

The mechanism of these lipid changes is of interest in understanding thyroid hormone actions or effects on energy metabolism. Among the parameters shown to affect biomembrane dependency are phospholipid:protein ratios (7), relative amounts of phospholipid polar groups (8), the degree of fatty acyl unsaturation $(4,5)$ and the cholesterol content (9). We compared the first three of these parameters in mitochondria from hypothyroid and control (euthyroid) rats. Because the pattern of unsaturated fatty acid abnormalities in mitochondria is consistent with a defect in $\Delta 5$ desaturation $(2,3)$, and because a decreased rate of overall fatty acyl desaturation has been demonstrated in the livers of hypothy roid rats (10), we also examined the fatty acyl contents of microsomal total lipids. If impaired hepatic desaturase activity accounts for the altered mitochondrial contents of 18:2 and 20:4 acyl groups, we expected the microsomal lipids to show a similar abnormality, such as the one reported in the nuclear envelope (11) that suggested a desaturation defect rather than a lesion specific for mitochondria. To test in vivo conversion of $18: 2$ to $20: 4$, which involves a $\Delta 6$-desaturation, an acyl chain elongation and the $\Delta 5$-desaturation, liver lipids were analyzed $6 \mathrm{hr}$ after injection of labeled linoleate. 


\section{MATERIALS AND METHODS}

Male rats weighing ca. $80 \mathrm{~g}$ were purchased thyroidectomized. The first series of control litter-mates and hypothyroid animals was obtained from Simonsen Laboratories, Inc., Gilroy, CA, and was maintained on a low-iodine, vitamin-enriched diet (Nutritional Biochemical Corp., Cleveland, $\mathrm{OH}$ ); the controls received $0.0005 \% \mathrm{KI}$ solution as drinking water, whereas the hypothyroid animals had $0.01 \% \mathrm{CaCl}_{2}$ in their water to prevent parathyroprivic tetany. Although growth retardation was evident in the hypothyroid group after 3 weeks, gas liquid chromatographic (GLC) analyses of mitochondrial phospholipid fatty acyl contents showed smaller abnormalities than those previously reported with hypothyroid rats additionally injected with $\mathrm{Na}^{131} \mathrm{I}(2,3)$. Further, experiments on the liver microsomal fatty acyl CoA $\Delta$ 9-desaturase showed that half of a group of rats made hypothyroid by surgery alone were able to induce the enzyme after a fasting-refeeding cycle, whereas none of those additionally pretreated with ${ }^{131} \mathbf{I}^{-}$could (12). We therefore report here the results comparing controls with thyroidectomized male rats (Spartan Res. Lab., Haslett, MI) treated as described plus an additional preliminary radiothyroidectomy through the ip injection of $0.5 \mathrm{mCi}$ of ${ }^{131} \mathrm{INa}$ a few days after surgery $(2,3)$. Animals were used for experiments at least later than 3 weeks after ${ }^{131} I^{-}$injection, and were allowed to feed ad lib up to the time of killing. One control and two hypothyroid animals were killed in each experiment to provide sufficient material for analyses.

After the rats were decapitated, livers were chilled in iced $0.25 \mathrm{M}$ sucrose and homogenized in a $10 \%$ suspension using a Teflon-glass tube. The dense pellet that sedimented at $600 \times \mathrm{g}$ for $5 \mathrm{~min}$ was removed and the mitochondria were sedimented at $10,000 \times \mathrm{g}$ for $10 \mathrm{~min}$, resuspended in iced $0.25 \mathrm{M}$ sucrose solution, and resedimented. Liver microsomes were prepared from a $10,000 \times \mathrm{g}$ supernatant, as described elsewhere (13). The microsomal pellet was gently resuspended with a Teflonglass homogenizer in $0.15 \mathrm{M}$ Tris acetate, $\mathrm{pH} 8.1$, leaving behind most of the glycogen (when present) and the microsomes were resedimented at $105,000 \times \mathrm{g}$ for $45 \mathrm{~min}$. The pellets were resuspended in cold $0.25 \mathrm{M}$ sucrose and diluted to $20 \mathrm{mg}$ protein $/ \mathrm{ml}$. Protein was determined using $0.16 \%$ deoxycholate to dissolve membrane-bound proteins, and bovine serum albumin was used as reference standard (14).

Lipids were extracted from freshly prepared mitochondrial suspensions with a 2:1 mixture of chloroform/methanol plus $0.005 \%$ butylated hydroxytoluene, using $20 \mathrm{ml} / \mathrm{ml}$ suspension. This mixture was homogenized for $1 \mathrm{~min}$ with a Tekmar high-speed tissue disintegrator and extracted according to Folch et al. (15). Neutral and polar lipids were separated on a silica gel column by elution with chloroform and methanol, respectively. Aliquots of total lipids and polar lipids were weighed after removal of solvents at $40 \mathrm{C}$ under $\mathrm{N}_{2}$, and at $23 \mathrm{C}$ under vacuum. Phospholipids were

TABLE 1

Lipids in Rat Liver Mitochondria

\begin{tabular}{ccc}
\hline & Controls (4) & Hypothyroids (6-9) \\
\hline Total Lipids $(\mu \mathrm{g} / \mathrm{mg}$ protein) & $0.14 \pm .02$ & $0.12 \pm .01$ \\
Phospholipids $(\mu \mathrm{g} / \mathrm{mg}$ protein) & $0.08 \pm .02$ & $0.07 \pm .01$ \\
Neutral lipids $(\mu \mathrm{g} / \mathrm{mg}$ protein) & $0.06 \pm .01$ & $0.05 \pm .007$ \\
Total lipid P $(\mu \mathrm{mol} / \mathrm{mg}$ protein) & $0.11 \pm .01$ & $0.11 \pm .02$ \\
Phospholipids (\% total phospholipid P) & & $47.7 \pm 2.9$ \\
PC & $55.4 \pm 3.8$ & $25.5 \pm 2.6$ \\
PE & $22.6 \pm 2.7$ & 20.5 \\
PS & $11.9 \pm 2.2$ & $6.5 \pm 1.2$ \\
\hline
\end{tabular}

$a_{p}<0.025$

Mitochondria were prepared, extracted and analyzed as described in Materials and Methods. Aliquots of total lipids, phospholipids and neutral lipids were dried and weighed. The P contents of total lipids and of the phospholipids resolved by TLC (PC = phosphatidylcholine; $\mathrm{PE}=$ phosphatidylethanolamine $\mathrm{CL}=$ cardiolipin, $\mathrm{PS}=$ phosphatidylserine and phosphatidylinositol) were measured. The numbers in parentheses after Controls and Hypothyroids denote how many separate measurements were done, each on materials from 1 control or 2 hy pothyroid animals. 
resolved on Silica Gel 60 TLC plates, developed in chloroform/methanol/glacial acetic acid $/ \mathrm{H}_{2} \mathrm{O}(100: 60: 16: 8, \mathrm{v} / \mathrm{v})$ according to Skipski et al. (16); locations were identified by very short exposure of the plates to $I_{2}$ vapor, after which the $I_{2}$ was removed under vacuum at $23 \mathrm{C}$. Four phospholipid classes were found: phosphatidylcholine (PC), phosphatidylethanolamine (PE), phosphatidylserine (PS; this component reacted with ninhydrin but probably also included phosphatidylinositol) and cardiolipin (CL). The TLC plates were scraped to remove each of the phospholipids, and total phosphorus was measured (17). In separate experiments, each phospholipid was dissolved in benzene, concentrated, and subjected to methanolysis for $45-60 \mathrm{~min}$ in methanol- $1 \% \mathrm{H}_{2} \mathrm{SO}_{4}$ at $70 \mathrm{C}$. Fatty acid methyl esters were measured by GLC (Varian Model 3700) using a $10 \%$ Silar $10 \mathrm{C}$ column with linear temperature programming, $170-200 \mathrm{C}$ at $3 \mathrm{C} / \mathrm{min}$ and a hydrogen flame ionization detector at $250 \mathrm{C}$. Results are given as area percentages.

To measure in vivo conversion of $18: 2$ to $20: 4$, rats were injected ip with $0.1 \mu \mathrm{Ci} / \mathrm{g}$ body wt of $1-{ }^{14} \mathrm{C}$-labeled linoleate $(57 \mathrm{Ci} /$ $\mathrm{mol}$; New England Nuclear) dissolved in bovine serum albumin, $24 \mathrm{mg} / \mathrm{ml}$. Animals were killed at $6 \mathrm{hr}$ and the livers were extracted as already described. Solvents were removed under $\mathrm{N}_{2}$ at $40 \mathrm{C}$. Aliquots of total lipids were: (a) assayed for ${ }^{14} \mathrm{C}$ by liquid scintillation counting; (b) resolved by TLC into neutral and polar lipid classes, which were counted; or (c) converted to fatty acid methyl esters and analyzed by a combined GLC and radio-assay instrument: a Packard Model 824 in combination with a proportional counter \#894 that gives simultaneous peaks for mass and radioactivity.

\section{RESULTS}

The low-iodine, vitamin-enriched diet fed to both control and hypothyroid rats contained $7.9 \mathrm{~g}$ of lipid/100 g dry wt. The fatty acid contents were: $4.5 \% 14: 0,17.2 \%$ $16: 0,0.5 \% 16: 1,9.1 \% 18: 0,39.3 \% 18: 1$, $28.8 \% 18: 2$ and $0.6 \% 18: 3$ fatty acids. The only significant source of $\omega 6$ fatty acids was thus $18: 2$; therefore, any $20: 4 \omega 6$ fatty acyl groups found in liver organelles must have been biosynthesized from linoleate.

The liver mitochondrial contents (on a wt/protein basis) of total extractable lipids, phospholipids and neutral lipids (by difference) were not significantly different from normal levels in hypothyroid rats (Table I). Total lipid phosphorus content/mg protein was identical in both groups, as well. However, the percentage contribution of each of the resolved phospholipid classes, as measured by percentage of total phospholipid $P$, showed that cardiolipin content was almost twice normal levels $(p<0.025)$ in the mitochondria of hypothyroid rats; although the contents of the other phospholipids were not significantly changed, PC and PS decreased slightly.

The fatty acyl contents of the combined and the resolved phospholipids are shown in Table II. The total phospholipids extracted from the liver mitochondria prepared from hypothyroid animals showed the abnormalities previously described $(2,3)$ : increased contents of $18: 2$ and 20:3 acyl groups (evidence in ref. 3 and comparison with

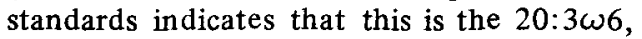
not the 20:3 $\omega 9$ isomer), decreased 20:4 acyl groups, decreases in the unsaturation index $(-11 \%)$ and a decreased ratio of 20:4/18:2. In addition, the current analyses showed a significant $31 \%$ increase in the proportion of 18:1 fatty acyl groups. Among the separated phospholipid classes, mitochondria from hypothyroid animals contained PC with even more strikingly elevated 18:2 and 20:3 and depressed 20:4 fatty acyl moieties, and an even lower 20:4/18:2 ratio, than appeared in the total phospholipids. The PE in the hypothyroids similarly contained excess proportions of 18:2 and 20:3 acyl groups, but the depletion of $20: 4$ acyl groups was less severe, although still significant. Stearic acid content was depressed. The PS(PI) fractions showed no significant fatty acyl group deviations from normal levels, but did reveal a decreased 20:4/18:2 ratio. Cardiolipins from control and hypothyroid preparations were similar in all fatty acyl residue contents.

Comparison of the fatty acyl group compositions of unresolved and resolved mitochondrial phospholipids in Table II indicates that some polyunsaturated acyl moieties were lost during the TLC separation. Using the percentage contribution of each of the phospholipid classes (Table I) together with their fatty acyl compositions (Table II), the calculated recovery of $18: 2$ acyl residues was $92.0 \%$ in control preparations and 104\% in hypothyroids. More 20:4 acyl groups were lost, but to a similar degree in controls (78.9\% recovery) and hypothyroids ( $79.2 \%$ recovery). Taken together with the similarity of the total phospholipid 


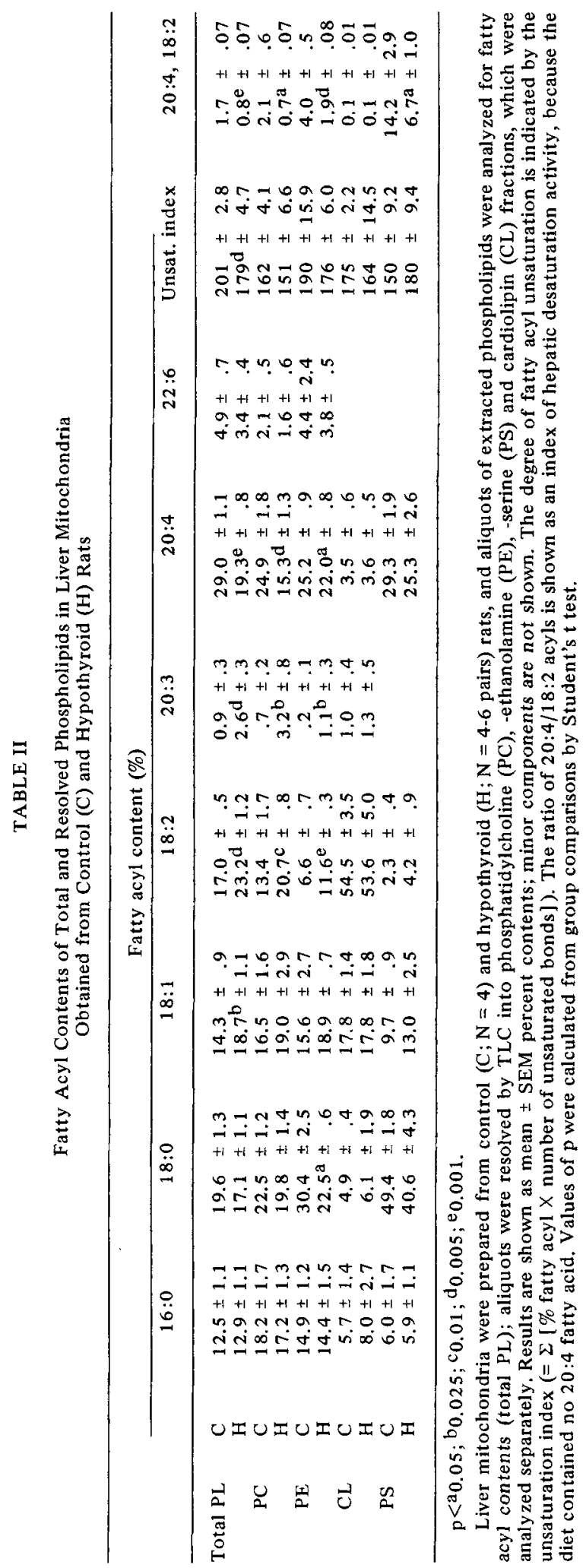

LIPIDS, VOL. 16, NO. 5 (1981) 
contents in both groups of animals (Table I), the fatty acyl contents of mitochondria prepared from the livers of hypothyroid rats were abnormally distributed.

Liver microsomal fatty acyl composition was measured in extracted total lipids (Table III). The distribution of the polyunsaturated fatty acyl groups in the microsomes from hypothyroid rats was much like that in the mitochondrial phospholipids, with increased $18: 2$ and $20: 3$, decreased $20: 4$ acyl residues, and decreased overall unsaturation index $(-11 \%)$. Because total microsomal protein, in $\mathrm{g}$ recovered/100 $\mathrm{g}$ wet wt of liver, was the same in hypothyroid rats $(0.85 \pm 0.10 \mathrm{SEM}$; $\mathrm{n}=9)$ and control rats $(0.88 \pm 0.12 \mathrm{SEM}$; $\mathrm{n}=8$ ), the fatty acyl contents of liver microsomes of hypothyroid rats were abnormally distributed. Microsomal lipids have not yet been further resolved for fatty acid analyses; however, we reported elsewhere (18) that hypothyroidism doubles the cholesterol content.

The excess 18:2 and the depleted 20:4 in liver mitochondria (Table II; refs. 2,3), microsomes (Table III) and nuclei (11) of hypothyroid rats point to a defective conversion of 18:2 to 20:4. To examine this hypothesis in vivo, we injected $\left[1-{ }^{14} \mathrm{C}\right]-$ linoleate into normal or hypothyroid rats and measured the hepatic incorporation and distribution of the label in lipids 6 hr later (Table IV). Hypothyroid rats had lower body weights and a lower liver:body-wt ratio than normals, but hypothyroid livers contained almost the normal proportion of lipid. The hypothyroid animals incorporated much less $(7-50 \%)$ of the label into liver lipids but, as in normals, the label appeared in phospholipids and triglycerides. Hypothy- roids converted much less $18: 2$ to the incorporated $20: 4$. While normal rats converted the $18: 2$ almost exclusively to $20: 4$, hypothyroid animals instead transformed large portions of the $1-{ }^{14} \mathrm{C}$ of $18: 2$ into saturated fatty acids; much more label was found in 16:0 and 18:0 than in 20:4. Taken together with the production of $20: 4$, the liver of the hypothyroid animal appeared to metabolize more of the $18: 2$ than did the liver of the normal rat.

\section{DISCUSSION}

The data obtained for hepatic organelle fatty acyl composition serve to eliminate some alternative mechanisms for the membrane abnormalities observed in hypothyroid rats. For example, the well known anorexia of hypothyroidism (19) might suggest a nutritional deficiency of essential fatty acids. Comparisons of food intake between controls and hypothyroids were not possible because the animals were fed ad lib. in open-bottomed cages. However, when hypothyroid rats were fasted $48 \mathrm{hr}$ and then given access to a $20 \%$ sucrose solution, they ingested as much sucrose/g body wt as did control animals; the hypothyroids failed to induce the microsomal $\Delta 9$-desaturase (12) but maintained the relative excess of linoleate in their liver mitochondria and microsomes either when they were refed sucrose or when they were just starved (F.L. Hoch and T.R. Chavis, unpublished data). These observations make dietary intake an unlikely cause of the results obtained here in the hypothyroid rats.

Because dietary $18: 2$ was the only source of the $\omega 6$ fatty acids in these rats, the

TABLE III

Fatty Acyl Contents of the Total Lipids in Liver Microsomes Prepared from Control and Hypothyroid Rats

\begin{tabular}{ccc}
\hline Fatty acyl & Controls (12) & Hypothyroids (12) \\
\hline $16: 0$ & $14.5 \pm .5$ & $15.6 \pm .6$ \\
$18: 0$ & $18.3 \pm .5$ & $18.7 \pm .7$ \\
$18: 1$ & $17.2 \pm .7$ & $17.6 \pm .6$ \\
$18: 2$ & $15.5 \pm .4$ & $19.6^{\mathrm{b}} \pm .7$ \\
$20: 3$ & $0.8 \pm .07$ & $2.3^{\mathrm{b}} \pm .2$ \\
$20: 4$ & $26.4 \pm .9$ & $17.3^{\mathrm{a}} \pm .8$ \\
$22: 6$ & $3.7 \pm .4$ & $3.9^{9} \pm .4$ \\
Unsaturation index & $191 \pm 2.3$ & $170^{\mathrm{b}} \pm 4.7$ \\
$20: 4 / 18: 2$ & $1.7 \pm .09$ & $0.9^{\mathrm{b}} \pm .06$ \\
\hline
\end{tabular}

$\mathrm{p}<\mathrm{a}^{\mathrm{a}} 0.005 ; \mathrm{b}_{0.001}$.

Liver microsomes were prepared, extracted and analyzed as described in Materials and Methods and in Table II. $\mathrm{N}$ is shown in parentheses. Mean \% of total fatty acids \pm SEM are shown. 
TABLE IV

Distribution of the $\left[1-{ }^{14} \mathrm{C}\right]$ of Labeled Linoleate in to Liver Lipids $6 \mathrm{hr}$ after Injection

\begin{tabular}{|c|c|c|c|c|c|}
\hline & \multicolumn{2}{|c|}{ Normal rats } & \multicolumn{3}{|c|}{ Hypothyroid rats } \\
\hline & $A$ & $\mathrm{~B}^{*}$ & $\mathrm{C}$ & $\mathrm{D}$ & $\mathbf{E}$ \\
\hline Body weight (g) & 344 & 345 & 175 & 191 & 170 \\
\hline Liver: body weight $(\mathrm{g} / 100 \mathrm{~g})$ & 3.8 & 3.7 & 2.8 & 2.7 & 3,1 \\
\hline Lipid: liver (mg/g) & 48.3 & 44.5 & 32.6 & 36.8 & 44.2 \\
\hline${ }^{14} \mathrm{C}$ in total lipid (dpm/g liver) & 256,782 & $143,261 *$ & 25,645 & 88,512 & 91,202 \\
\hline $\begin{array}{l}\text { (\% of dose) } \\
{ }^{14} \mathrm{C} \text { in phospholinids }\end{array}$ & 4.53 & 2.49 & 0.33 & 1.10 & 1.32 \\
\hline $\begin{array}{l}\text { (\% of total lipid dpm) } \\
{ }^{14} \mathrm{C} \text { in triglycerides }\end{array}$ & 63.9 & 62.3 & 73.2 & 79.4 & 74.0 \\
\hline $\begin{array}{l}\text { (\% of total lipid dpm) } \\
{ }^{14} \mathrm{C} \text { in fatty acid methyl esters } \\
\text { of total lipids } \\
\text { (\% of total lipid } \mathrm{dpm})\end{array}$ & 32.9 & 34.5 & 24.8 & 19.1 & 24.0 \\
\hline $16: 0$ & 0 & 9.4 & 43.9 & 14.3 & 24.1 \\
\hline $18: 0$ & 0 & 0 & 13.3 & 8.0 & 2.4 \\
\hline $18: 2$ & 74.8 & 66.0 & 42.8 & 65.8 & 59.1 \\
\hline $20: 4$ & 25.2 & 24.6 & 0 & 11.8 & 14.3 \\
\hline
\end{tabular}

Rats were injected intraperitoneally with $\left[1{ }^{14} \mathrm{C}\right]$ linoleate, $0.10 \mu \mathrm{Ci} / \mathrm{g}$ body wt (except for normal rat $\mathrm{B}^{*}$, which received $0.022 \mu \mathrm{Ci} / \mathrm{g}$; corrected values for $\mathrm{dpm} / \mathrm{g}$ liver are shown) and killed 6 hr later. Extracted total lipids of the livers were counted; aliquots were resolved by TLC, or subjected to methanolysis and resolved by GLC combined with radio-assay of the fatty acid methyl esters.

concomitant depletion of membrane 20:4 acyl groups implicates a defective biosynthesis from 18:2, or, given the adequacy of this conversion, an increase in the amount of phospholipids that are normally selectively acylated to favor $18: 2$ rather than $20: 4$ acyl groups. The latter process normally occurs in the synthesis of cardiolipins, which contain $55 \% 18: 2$ and only $6 \% 20: 4$ acyl groups, as compared to the 5-10\% 18:2 and the $25 \%$ of $20: 4$ acyl residues found in the other mitochondrial phospholipid classes (Table II). An increase in the relative amount of $\mathrm{CL}$ would in this way contribute to the hypothyroid fatty acyl pattern, and this is what was observed (Table I); further, the CL fatty acyl content of hypothyroid mitochondria resembled that in controls. However, even in the hypothyroid mitochondrial membranes, cardiolipin fatty acids make only a minor contribution to the overall fatty acid distribution. In addition, $\mathrm{CL}$ is mostly a mitochondrial component (20) and so would not be significantly involved in the similarly abnormal fatty acyl compositions of microsomes (Table III) and nuclei (11). In liver mitochondria of hypothyroid rats, as in controls, PC and PE represented $75 \%$ of the phospholipid content (Table I), and their abnormally distributed polyunsaturated fatty acids predominated in producing the overall pattern (Table II). The severity of the abnormal distribution appeared in the order $\mathrm{PC}>\mathrm{PE}>\mathrm{PS}(\mathrm{PI})>\mathrm{CL}$. The exchange of the PL classes follows this order, with $\mathrm{PC}>$ $P E$ and $C L$ not exchanging at all (21). In contrast, the rapid replacement of $P L$, as measured by the appearance of injected labeled phosphate in hepatic organelle membranes, is faster in PE than in PC (22).

The observation that, in hypothyroid rats, the liver microsomes contained polyunsaturated fatty acyl residues that were distributed (Table III) in the same pattern that was seen in the liver mitochondria (Table II; refs. 2,3) and nuclei (11) suggests that this general pattern reflects a polyunsaturated fatty acid pool accessible to organelle membranes through acylationdeacylation reactions (23). It makes unlikely the alternative that the mitochondrion, which appears to possess some degree of autonomy in forming its own membrane phospholipids, as it does with polypeptide synthesis (22), is the source of the lipid defects. This conclusion is supported by observations that liver mitochondrial contributions to the synthesis of unsaturated fatty acids, i.e., the chain elongation, are insensitive to the hypothyroid state (10).

The diminished ability of hypothyroid rats to incorporate the label of $\left[1-{ }^{14} \mathrm{C}\right] 18: 2$ into liver lipids (Table IV) may be connected with their decreased liver coenzyme A 
concentrations (24). Little, if any, label was incorporated into saturated fatty acyl groups by normal rats under our conditions, whereas a major portion was incorporated by the hypothyroid animals. This excessive conversion appears surprising, as did Gordon and Goldberg's (25) observation that hypothyroid humans convert labeled 16:0 to $\mathrm{CO}_{2}$ faster than controls, in view of the decreased fatty acid oxidation and high $R Q$ in this condition. Gordon and Goldberg suggested that diminution and slowed turnover of the endogenous "active" FFA pool minimally dilutes administered fatty acid and that absolute rates of conversion are not necessarily increased. This explanation seems applicable to our data on $18: 2$ and to the apparent anomalies of absolute accumulation of 18:2 in liver lipids (Tables II and III), and depressed fatty acid synthesis in hypothy roidism (26). Theoretically, two alternatives might explain the pattern of label distribution in hypothyroidism: either defective conversion of $18: 2$ to $20: 4$ diverts the label toward acetyl CoA and 16:0 (and perhaps $\mathrm{CO}_{2}$, which we did not measure), or accelerated $\beta$-oxidation of $18: 2$ to acetyl CoA competes with the conversion of $18: 2$ to 20:4. However, many observations since 1928 (27) have confirmed that $\beta$-oxidation is slowed in hypothyroidism, not accelerated.

Therefore, the most likely mechanism for the observed alterations of hepatic organelle membrane fatty acyl composition in hypothyroidism is a defect in the microsomal conversion of $18: 2 \omega 6$ to $20: 4 \omega 6$ fatty acyls, which involves a chain elongation and $\Delta 6$ - and $\Delta 5$-desaturations. Hypothyroidism has been reported to slow microsomal chain elongation by $-35 \%(10)$, although others find no change (28). However, the elongation 18:3 $\omega 6 \rightarrow 20: 3 \omega 6$ normally proceeds 5.6 times faster than the $\Delta 5$-desaturation and 4.5 times faster than the $\Delta 6$-desaturation (29), and so would not seem to be ratecontrolling in any case. Preliminary data on hypothyroid rats show decreased liver microsomal $\Delta 6-$ and $\Delta 5$-desaturase $V$ (maximal velocity, determined by extrapolation to infinite [S]) and unchanged $\mathrm{Km}$ (18); the $\Delta 6$-desaturase $\mathrm{V}$ but not the $\mathrm{Km}$ rises, and liver microsomal 20:4 acyl content falls within $4 \mathrm{hr}$ after injection of L-triiodothyronine; $\Delta 5$-desaturase activity has not yet been measured this early after hormone injection.

deGomez Dumm et al. (28) report that hypothyroidism (using female rats fed propylthiouracil) significantly decreases the
18:2 content of liver fatty acids and does not alter either the $20: 4$ content or the microsomal $\Delta 9$ - or $\Delta 6$-desaturase activities. Their data on fatty acid composition disagree with Ellefson and Mason's (30) on liver total lipids and ours (Tables II, III; refs. 2,3 ) on liver organelle fatty acids. We agree that hypothyroidism leaves the $\Delta 9$-desaturase activity unchanged but find that it blocks the normal dietary induction of this activity (12). As already noted, we find decreased $\Delta 6$-desaturase activity in hypothyroid rats. Presumably, the different in vivo and in vitro experimental conditions account for these apparent divergencies.

\section{ACKNOWLEDGMENTS}

These studies were supported by an NSF grant (GB-42256) to F. L. Hoch.

\section{REFERENCES}

1. Chen, Y.-D.I., Fed. Proc. 34:314 (1975).

2. Chen, Y.-D.I., and F.L. Hoch, Arch. Biochem. Biophys. 172:741 (1976).

3. Chen, Y.-D.I., and F.L. Hoch, Arch. Biochem. Biophys. $181: 470$ (1977).

4. Raison, J.K., Bioenergetics 4:285 (1973).

5. McMurchie, E.J., and J.K. Raison, Biochim. Biophys. Acta 554:364 (1979).

6. Hulbert, A.J., M.L. Augee and J.K. Raison, Biochim. Biophys. Acta 455:597 (1976).

7. Thompson, J.F, D.A. Habeck, S.L. Nance and K.L. Beetham, J. Cell Biol, $41: 312$ (1969).

8. Papahadjopoulos, D., J. Colloid Sci. 58:459 (1979).

9. Kimelberg, H.K., Biochim. Biophys. Acta 413 : 143 (1975).

10. Landriscina, G., G.V. Gnoni and E. Quagliariello, Eur. J. Biochem. 71:135 (1976).

11. Shaw, M.J., and F.L. Hoch, Life Sci. 19:1359 (1976).

12. Hoch, F.L., J.W. DePierre and L. Ernster, Eur. J. Biochem. 109:301 (1980).

13. Ernster, L., P. Siekevitz and G.E. Palade, J. Cell Biol. 15:541 (1962).

14. Gornall, A.G., C.J. Bardawill and M.W. David, J. Biol. Chem. 177:751 (1949).

15. Folch, J., M. Lees and G.H. Sloane-Stanley, J. Biol. Chem. 226:497 (1957).

16. Skipski, V.P., R.F. Peterson and M. Barclay, Biochem. J. $90: 374$ (1964).

17. Bartlett, G.R., J. Biol. Chem. 234:466 (1959).

18. Hoch, F.L., in "Endocrinology 1980," Proc. 6th Intl. Conf. Endocrinol. Melbourne, Australia Feb. $10: 16,1980$, edited by I.A. Cumming, J.W. Funder and F.A.O. Mendelsohn, Austral. Acad. Sci. Canberra, 1980, pp. 409-412.

19. Means, J.H., L.J. DeGroot and J.B. Stanbury, in "The Thyroid and its Diseases," 3rd Ed., McGraw Hill, New York, 1963.

20. Getz, G.S., W. Bartley, D. Lurie and B.M. Notton, Biochim. Biophys, Acta 152:325 (1968).

21. Wirtz, K.W.A., and D.B. Zilversmit, J. Biol. Chem. 243:3596 (1968).

22. McMurray, W.C., in "Form and Function of Phospholipids," 2nd Ed., B.B.A. Library 3, 
edited by G.B. Ansell, J.N. Hawthorne and R.M.C. Dawson; Elsevier Scientific Publishing Co., New York, 1973, p. 205.

23. Reitz, R.C., M. El-Sheikh, W.E.M. Lands, I.A Ismail and F.E. Gunstone, Biochim. Biophys. Acta 176:480 (1969).

24. Tabachnick, I.I.A., and D.D. Bonnycastle, J Biol. Chem. 207:757 (1954).

25. Gordon, E.S., and M. Goldberg, Metabolism 13:591 (1964).

26. Dayton, S., J. Dayton, F. Drimmer and F.E. Kendall, Am. J. Physiol. 199:71 (1960).
27. Abelin, I., and P. Kürsteiner, Biochem. Z. 198:19 (1928).

28. de Gomez Dumm, I.N.T., M.J.T. deAlaniz and R.R. Brenner, Adv. Exp. Med. Biol. 83:609 (1977).

29. Bernert, J.T., Jr., and H. Sprecher, Biochim. Biophys. Acta 398:354 (1975).

30. Ellefson, R.D., and H.L. Mason, Endocrinology 75:179 (1964).

[Received October 3, 1981] 